\title{
Lichenicolous fungi from Russia, mainly from the Magadan Region
}

\author{
Mikhail P. Zhurbenko' ${ }^{1}$ \& Elena V. Zheludeva ${ }^{2}$ \\ ${ }^{1}$ Laboratory of the Systematics and Geography of Fungi, Komarov Botanical Institute, Russian Academy of Sciences, \\ Professora Popova str. 2, St. Petersburg, 197376, Russia. E-mail: zhurb58@gmail.com \\ ${ }^{2}$ Laboratory of botany, Institute of Biological Problems of North, Russian Academy of Sciences, Portovaya street 18, \\ Magadan, 685000, Russia. E-mail: elena.zheludeva.88@mail.ru
}

\begin{abstract}
Twenty two species of lichenicolous fungi are reported, 19 of which are new to Magadan Region of Russia. Spirographa fusisporella is new to Asia. Sphaerellothecium cf. parmeliae growing on species of Parmelia s. str. and "Sphaeropezia" sp. on Hypogymnia physodes are briefly described, illustrated and discussed.
\end{abstract}

Keywords: lichen-inhabiting fungi, biogeography, taxonomy, ecology, Beringia, Northern Asia

\section{INTRODUCTION}

Despite the relatively well-studied lichenicolous mycobiota of Chukotka (Kristinsson et al., 2010), the Magadan Region next to it still remained virtually unexplored in this regard. So far just nine species of lichenicolous fungi have been reported from the region, viz. Echinodiscus kozhevnikovii Zhurb. (on Cetraria laevigata), Endococcus nanellus Ohlert (on Stereocaulon alpinum), Lichenostigma maureri Hafellner (on Alectoria ochroleuca), Phaeospora arctica Horáková \& Alstrup (on Cetraria islandica), Sphaerellothecium minutum Hafellner (on Sphaerophorus fragilis), S. reticulatum (Zopf) Etayo [uncertain report on Arctoparmelia separata possibly referring to "Lichenostigma arctoparmeliae" R. Sant. (ined.)], Stigmidium hafellneri Zhurb. (on Flavocetraria cucullata), Thamnogalla crombiei (Mudd) D. Hawksw. (on Thamnolia vermicularis) and Zwackhiomyces sipmanii Diederich \& Zhurb. (on Phaeorrhiza sareptana var. sphaerocarpa) (Zhurbenko, 2008, 2009a, b, 2010, 2012; Diederich $\&$ Zhurbenko, 2009; Zhurbenko \& Vershinina, 2014). This paper presents results of a revision of a lichen collection mainly from Magadan Region of Russia, including 19 species new to the region and some other noteworthy findings.

\section{MATERIAL AND METHODS}

The material was identified by the first author using Zeiss microscopes Stemi 2000-CS and Axio Imager A1 equipped with Nomarski differential interference contrast (DIC) optics. Microscopical examination was done in water, $10 \% \mathrm{KOH}(\mathrm{K})$, Lugol's iodine directly (I) or after a $\mathrm{KOH}$ pre-treatment (K/I) or brilliant cresyl blue $(\mathrm{BCr})$. The ascomatal diameter and the length, breadth and length/breadth ratio $(1 / b)$ of asci and ascospores (when $n>10$ ) are given as: $(\min -) \bar{X}-\mathrm{SD}-\overline{\mathrm{X}}+\mathrm{SD}(-\max )$, where min and $\max$ are the extreme values, $\bar{X}$ the arithmetic mean, and SD the corresponding standard deviation. Measurements were taken from water mounts, unless otherwise indicated. The nomenclature of the host lichens follows Esslinger (2015). Examined specimens are deposited in the mycological herbarium of the V. L. Komarov Botanical Institute in St.-Petersburg, Russia (LE).

\section{THE SPECIES}

Species new to Magadan Region of Russia are denoted by an asterisk (*).

*Abrothallus parmeliarum (Sommerf.) Arnold - Magadan Region, Ola District, Taui River, ichthyological field station, $59^{\circ} 47^{\prime} \mathrm{N}, 148^{\circ} 16^{\prime} \mathrm{E}$, alt. $35 \mathrm{~m}$, mixed Larix forest, on Parmelia sulcata (lobes) growing on Larix, 18.06.2011, E. V. Zheludeva (LE 261202, LE 261281a); same district, Magadan Reserve, $7 \mathrm{~km}$ downstream by Yama River from Studenaya cabin, $59^{\circ} 44^{\prime} \mathrm{N}$, $153^{\circ} 40^{\prime} \mathrm{E}$, alt. $60 \mathrm{~m}$, forested stone field, on P. omphalodes (thallus), 24.07.2010, E. V. Zheludeva (LE 264309a).

Note - Immature hyaline spores often have a distinct thick halo which is reduced at maturity.

*Ameroconium cladoniae U. Braun \& Zhurb. - Magadan Region, Ola District, Zavyalova Is- 
land, Rassvet Bay, $59^{\circ} 04^{\prime} \mathrm{N}, 150^{\circ} 38^{\prime} \mathrm{E}$, alt. 45 $\mathrm{m}$, Betula ermanii forest, on Cladonia coccifera agg. (podetia, basal squamules), 29.06.2010, E. V. Zheludeva (LE 264269).

Note - This recently described species was formerly known only from Irkutsk Region and Trans-Baikal Territory in Russia from Cladonia alaskana, C. arbuscula and C. rangiferina (Zhurbenko \& Braun, 2013; Zhurbenko \& Yakovchenko, 2014). Cladonia coccifera agg. is a new host species.

*Epicladonia sandstedei (Zopf) D. Hawksw. Magadan Region, North-Even District, mouth of Nayakhan River, $61^{\circ} 55^{\prime} \mathrm{N}, 159^{\circ} 00^{\prime} \mathrm{E}$, alt. $5 \mathrm{~m}$, on Cladonia cornuta (podetia), 14.06.2008, E. V. Zheludeva (LE 264349).

Note - Formerly known in Asian Russia from Karachaevo-Cherkesiya Republic, Krasnoyarsk Territory and Trans-Baikal Territory (Zhurbenko, 1998; Zhurbenko \& Kobzeva, 2014; Zhurbenko \& Yakovchenko, 2014).

*Evernitcola flexispora D. Hawksw. - Magadan Region, Ola District, Taui River, ichthyological field station, $59^{\circ} 47^{\prime} \mathrm{N}, 148^{\circ} 16^{\prime} \mathrm{E}$, alt. $40 \mathrm{~m}$, Pinus pumila shrubs, on Nephroma arcticum (lobes), 23.06.2009, E. V. Zheludeva (LE 261323).

*Lichenoconium lecanorae (Jaap) D. Hawksw. Magadan Region, North-Even District, Arestovo settlement at Gizhiga River, $61^{\circ} 58^{\prime} \mathrm{N}, 160^{\circ} 26^{\prime} \mathrm{E}$, alt. $30 \mathrm{~m}$, Pinus-Betula nana vegetation, on Flavocetraria cucullata (lobes), 9.07.2008, E. V. Zheludeva (LE 261313); same region, Ola District, Magadan Reserve, $7 \mathrm{~km}$ downstream by Yama River from Studenaya cabin, $59^{\circ} 45^{\prime} \mathrm{N}$, $153^{\circ} 34^{\prime} \mathrm{E}$, alt. $70 \mathrm{~m}$, Chosenia-Larix forest, on Melanohalea olivacea (damaged hymenium of apothecia), 17.07.2010, E. V. Zheludeva (LE 261461b).

Note - Flavocetraria is a new host genus.

*Lichenoconium usneae (Anzi) D. Hawksw. Magadan Region, Ola District, Taui River, ichthyological field station, $59^{\circ} 47^{\prime} \mathrm{N}, 148^{\circ} 16^{\prime} \mathrm{E}$, alt. $35 \mathrm{~m}$, Larix-Betula forest, on Physcia aipolia (destroyed apothecia) growing on Betula, 19.05.2009, E. V. Zheludeva (LE 264369b).

*Lichenopeltella Cetrarilcola (Nyl.) R. Sant. - Chukotka Autonomous Area, Anadyr Dis- trict, Emeem Lagoon, Malyi Arinai Mountain, $62^{\circ} 40^{\prime} 53.90^{\prime \prime} \mathrm{N}, 179^{\circ} 21^{\prime} 6.00^{\prime} \mathrm{E}$, alt. $95 \mathrm{~m}$, dwarf shrub-lichen tundra, on Arctocetraria andrejevii (lobe bases), 6.08.2012, D. S. Lysenko (LE 261433); Magadan Region, Tenkinskii District, bank of Omchak River, $61^{\circ} 33^{\prime} \mathrm{N}, 147^{\circ} 57^{\prime} \mathrm{E}$, alt. $860 \mathrm{~m}$, Larix-Pinus pumila vegetation, on $\mathrm{Ce}$ traria islandica (lobe bases), 30.06.2012, E. V. Zheludeva (LE 264469).

Notes - Asci 29-41(-48) × 10-12(-13) $\mu \mathrm{m}(\mathrm{n}=$ 10 , in $\mathrm{K})$, 4(-6)-spored. Ascospores (11.0-)13.6$17.4(-19.1) \times(2.8-) 3.1-3.5(-3.8) \mu \mathrm{m}, 1 / \mathrm{b}=(3.2-)$ 4.1-5.3(-6.1) $(\mathrm{n}=32$, in $\mathrm{K})$, usually with 2 large guttules in each cell. Hawksworth (1980) gave sizes of asci as 25-35 $\times 8-12 \mu \mathrm{m}$ and ascospores $14-16 \times 2.5-4 \mu \mathrm{m}$. Formerly known in Russia from Irkutsk Region and Buryatia Republic (Zhurbenko \& Vershinina, 2014). The species has mostly been collected on Cetraria islandica, but has also been reported from Arctocetraria andrejevii (Alstrup et al., 2009).

*Lichenostigma Alpinum (R. Sant., Alstrup \& D. Hawksw.) Ertz \& Diederich - Magadan Region, North-Even District, Arestovo settlement at Gizhiga River, $61^{\circ} 58^{\prime} \mathrm{N}, 160^{\circ} 26^{\prime} \mathrm{E}$, alt. $15 \mathrm{~m}$, on Ochrolechia frigida (thallus), 9.07.2008, E. V. Zheludeva (LE 264459).

*Lichenostigma cosmopolites Hafellner \& Calat. - Magadan Region, Magadan, Nagaeva Bay, Morportovskaya Mountain, 59 $34^{\prime} \mathrm{N}, 150^{\circ} 38^{\prime} \mathrm{E}$, alt. $150 \mathrm{~m}$, talus with Betula ermanii, on Xanthoparmelia stenophylla (disc of apothecia, thallus) growing on stone, 26.06.2007, E. V. Zheludeva (LE 264419).

Note - Formerly known in Asian Russia from Karachayevo-Circassian Republic, Trans-Baikal Territory and Jewish Autonomous Region (Zhurbenko, 2014; Zhurbenko \& Kobzeva, 2014; Zhurbenko \& Yakovchenko, 2014).

LiCHENOSTIGMA MAURERI Hafellner (anamorph) - Magadan Region, Magadan, Nagaeva Bay, Morportovskaya Mountain, 59 $34^{\prime} \mathrm{N}, 150^{\circ} 38^{\prime} \mathrm{E}$, alt. $310 \mathrm{~m}$, sparse Larix-Pinus pumila vegetation, on Alectoria lata (thallus) growing on Larix, 5.07.2005, E. V. Zheludeva (LE 261252).

Notes - Formerly reported in Magadan Region also from the vicinities of Magadan (Zhurbenko, 2009b). Alectoria lata is a new host species. 
*Nesolechia oxyspora (Tul.) A. Massal. - Magadan Region, Ola District, Taui River, ichthyological field station, $59^{\circ} 47^{\prime} \mathrm{N}, 148^{\circ} 16^{\prime} \mathrm{E}$, alt. $35 \mathrm{~m}$, mixed Larix forest, on Parmelia sulcata (thallus), 18.06.2011, E. V. Zheludeva (LE 261281b); 20.05.2009, E. V. Zheludeva (LE 264499); same district, Magadan Reserve, $7 \mathrm{~km}$ downstream by Yama River from Studenaya cabin, $59^{\circ} 44^{\prime} \mathrm{N}$, $153^{\circ} 40^{\prime} \mathrm{E}$, alt. $60 \mathrm{~m}$, forested stone field, on $P$. omphalodes (thallus), 24.07.2010, E. V. Zheludeva (LE 264309c).

*Plectocarpon linitae (R. Sant.) Wedin \& Hafellner - Magadan Region, Ola District, Zavyalova Island, Rassvet Bay, $59^{\circ} 04^{\prime} \mathrm{N}, 150^{\circ} 38^{\prime} \mathrm{E}$, alt. $80 \mathrm{~m}$, Betula ermanii-Alnus forest, on Lobaria linita (thallus), 20.06.2010, E. V. Zheludeva (LE 261353).

*Roselliniella cladoniae (Anzi) Matzer \& Hafellner - Magadan Region, North-Even District, Arestovo settlement at Gizhiga River, $61^{\circ} 58^{\prime} \mathrm{N}$, $160^{\circ} 26^{\prime} \mathrm{E}$, alt. $15 \mathrm{~m}, 9.07 .2008$, E. V. Zheludeva, on Cladonia sulphurina (dark basal parts of podetia) (LE 261443a), on C. coccifera agg. (dark basal parts of podetia) (LE 261443b).

*SAgediopsis campsteriana (Linds.) D. Hawksw. $\&$ R. Sant. - Magadan Region, Ola District, Zavyalova Island, near lighthouse at Rassvet Bay, $59^{\circ} 00^{\prime} \mathrm{N}, 150^{\circ} 29^{\prime} \mathrm{E}$, alt. $390 \mathrm{~m}$, stony lichen tundra, on Ochrolechia frigida (disc and margin of apothecia, thallus), 17.08.2009, E. V. Zheludeva (LE 264259).

Notes - Ascomata wall brown throughout or in young ascomata the outer layer reddish brown; pigmentation patchy in cross section. Formerly known in Asian Russia from Yamal-Nenets Autonomous Area, Krasnoyarsk Territory and Chukotka Autonomous Area (Zhurbenko, 2009c).

*SPhaERELlothecium Cladonitcola E.S. Hansen \& Alstrup - Magadan Region, Tenkinskii District, Rodionovskii Creek (tribute of Nelkoba River), $61^{\circ} 22^{\prime} \mathrm{N}, 148^{\circ} 41^{\prime} \mathrm{E}$, alt. $560 \mathrm{~m}$, Pinus pumila vegetation, on Cladonia arbuscula (podetia), 4.07.2012, E. V. Zheludeva (LE 261212).

Notes - Examined material fits the species protologue (Hansen \& Alstrup, 1995), except that ascomata are smaller, 25-35(-45) $\mu \mathrm{m}(\mathrm{n}=25)$ vs. 30-60 $\mu \mathrm{m}$ diam. Wall of asci and ascospores $\mathrm{BCr}-$. Formerly known in Asia and Russia only from Chukotka (Hansen \& Alstrup, 1995).
SPHAERELlothecium Minutum Hafellner - Magadan Region, Ola District, Magadan Reserve, Yama River, $59^{\circ} 44^{\prime} \mathrm{N}, 153^{\circ} 40^{\prime} \mathrm{E}$, alt. $60 \mathrm{~m}$, forested stone field, on Sphaerophorus globosus, 24.07.2010, E. V. Zheludeva (LE 264449a).

Note - Formerly known in Magadan Region from the vicinities of Magadan (Zhurbenko, 2008).

*Sphaerellothecium cf. PARmeliae Diederich \& Etayo - Russia: Murmansk Region, near Alakurtti settlement, $66^{\circ} 58^{\prime} \mathrm{N}, 30^{\circ} 20^{\prime} \mathrm{E}$, alt. $190 \mathrm{~m}$, Betula forest, on Parmelia saxatilis, 5.07.1972, A. V. Dombrovskaya (LE 264409); Komi Republic, Northern Ural, headwaters of Pechora River, Yanypupuner Range, Mt. "981", $62^{\circ} 05^{\prime} \mathrm{N}, 59^{\circ} 06^{\prime} \mathrm{E}$, alt. $800 \mathrm{~m}$, mountain tundra, on $P$. saxatilis, 30.06.1997, M. P. Zhurbenko 97220 (LE 210213); Krasnoyarsk Territory, Taimyr Peninsula, Bikada River, $74^{\circ} 50^{\prime} \mathrm{N}, 106^{\circ} 30^{\prime} \mathrm{E}$, tundra, 07.1989, E. B. Pospelova [LE 233398; published as S. parmeliae (Zhurbenko, 2009a)]; Republic of Tuva, Big Yenisey River valley, near Toora-Khem settlement, $52^{\circ} 26^{`} \mathrm{~N}, 96^{\circ} 05^{`} \mathrm{E}$, alt. $850 \mathrm{~m}$, Larix forest, on P. omphalodes, 29.07.1995, T. N. Otnyukova (LE 207772); Trans-Baikal Territory, Sokhondinskii Reserve, Agutsa River at $1 \mathrm{~km} \mathrm{~N}$ of Buninda Cabin, 4942'59" N, $111^{\circ} 22^{\prime} 50$ " E, alt. 1180 m, BetulaLarix forest, on P. omphalodes, 22.07.2008, L. S. Yakovchenko (LE 264479); Magadan Region, Ola District, Magadan Reserve, Yama River, $59^{\circ} 45^{\prime} \mathrm{N}, 153^{\circ} 35^{\prime} \mathrm{E}$, alt. $70 \mathrm{~m}$, mixed Larix forest, on P. sulcata, 23.07.2010, E. V. Zheludeva (LE 261363); same reserve, $7 \mathrm{~km}$ downstream by Yama River from Studenaya cabin, $59^{\circ} 44^{\prime} \mathrm{N}$, $153^{\circ} 40^{\prime} \mathrm{E}$, alt. $60 \mathrm{~m}$, forested stone field, on P. omphalodes, 24.07.2010, E. V. Zheludeva (LE 264309b); Chukotka Autonomous Area, Rauchua River, near abandoned Baranikha settlement, $68^{\circ} 33^{\prime} \mathrm{N}, 168^{\circ} 12^{\prime} \mathrm{E}$, stony tundra, on P. omphalodes, 20.06.1971, I. I. Makarova (LE 264299); same autonomous area, near confluence of Enmyvaam and Shustraya Rivers, $68^{\circ} 15^{\prime} \mathrm{N}, 166^{\circ} 03^{\prime} \mathrm{E}$, Pinus pumila shrubs and scree tundra, on P. omphalodes, 30.06.1980, I. I. Makarova (LE 261373).

Specimen of an externally typical Sphaerellothecium parmeliae examined for comparison: Russia, Murmansk Region, Barents Sea coast, Shel'pino Bay, $69^{\circ} 05^{\prime} \mathrm{N}, 36^{\circ} 12^{\prime} \mathrm{E}$, alt. $10 \mathrm{~m}$, coastal cliffs among tundra, on Parmelia omphalodes (thallus), 4.09. 1997, M. P. Zhurbenko 97344 (LE 233170). 
Notes - Vegetative hyphae branched, forming a dense superficial reticulum, medium brown, 2.5-4(-5) $\mu \mathrm{m}$ thick, with scaly fissured surface. Ascomata black, glossy, subglobose, (25-)40$60(-65) \mu \mathrm{m}$ diam. $(\mathrm{n}=25)$, with ostiole ca. $5-8$ $\mu \mathrm{m}$, semi-immersed to superficial, dispersed. Asci saccate, ovoid or pyriform, 21-30(-37) $\times$ (11-)12-14(-17) $\mu \mathrm{m}(\mathrm{n}=16), 8$-spored, wall BCr-. Ascospores narrowly ellipsoid with wider upper cell (obskittle-shaped), hyaline, (8.4-)9.3-11.1($12.5) \times(3.0-) 3.2-4.0(-4.5) \mu \mathrm{m}, 1 / \mathrm{b}=(2.1-) 2.5-$ $3.1(-3.4)(n=54), 1$-septate, sometimes slightly constricted at the septum, smooth-walled, nonhalonate, wall $\mathrm{BCr}-$. Infections usually dispersed over healthy-looking host lobes, but occasionally also growing on bleached or darkened areas of the lobes (Fig. 1A, B).

In the protologue of Sphaerellothecium parmeliae (Etayo \& Diederich, 1998) its vegetative hyphae were reported being smooth-walled, ascomata somewhat smaller, viz. 25-40(-60) $\mu \mathrm{m}$ diam., asci smaller, 19-23 × 9-12.5 $\mu \mathrm{m}$, ascospores oval and smaller, 8.5-10 $\times 3-4 \mu \mathrm{m}$. The fungus was also characterized by its infections arising from black necrotic areas of the host lobes. However, examined material of an externally typical $S$. parmeliae, strictly associated with well delimited black areas of the host thalli (Fig. 1C), has ascomata [(30-)40-50(-70) $\mu \mathrm{m}$ diam. $(\mathrm{n}=62)]$ and ascospores $[(9.7-) 10.3-$ $11.9(-13.0) \times(4.0-) 4.2-4.8(-5.3) \mu \mathrm{m} 1 / \mathrm{b}=$ (2.1-)2.3-2.7(-2.9) ( $\mathrm{n}=43$, in $\mathrm{BCr}$ or water)] similar in size to those of $S$. cf. parmeliae. It is could be hypothesized that the species is not obligately associated with black necrotic areas of the host thallus, which might have an independent origin.

Sphaerellothecium parmeliae was formerly reported in Russia from Murmansk Region and Krasnoyarsk Territory (Zhurbenko \& Otnyukova, 2001; Zhurbenko, 2009a). The species is so far known in Asia only from Russia and Turkey (Hafellner \& John, 2006).

*Sphaerellothecium sp. - Magadan Region, Magadan, Nagaeva Bay, Morportovskaya Mountain, $59^{\circ} 34^{\prime} \mathrm{N}, 150^{\circ} 38^{\prime} \mathrm{E}$, alt. $310 \mathrm{~m}$, sparse LarixPinus pumila vegetation, on Arctoparmelia centrifuga (thallus), 5.07.2005, E. V. Zheludeva (LE 261262); same region, Ola District, Zavyalova Island, Rassvet Bay, $59^{\circ} 04^{\prime} \mathrm{N}, 150^{\circ} 38^{\prime} \mathrm{E}$, alt. 25 $\mathrm{m}$, talus, on A. centrifuga (thallus), 13.08.2009, E. V. Zheludeva (LE 261233).

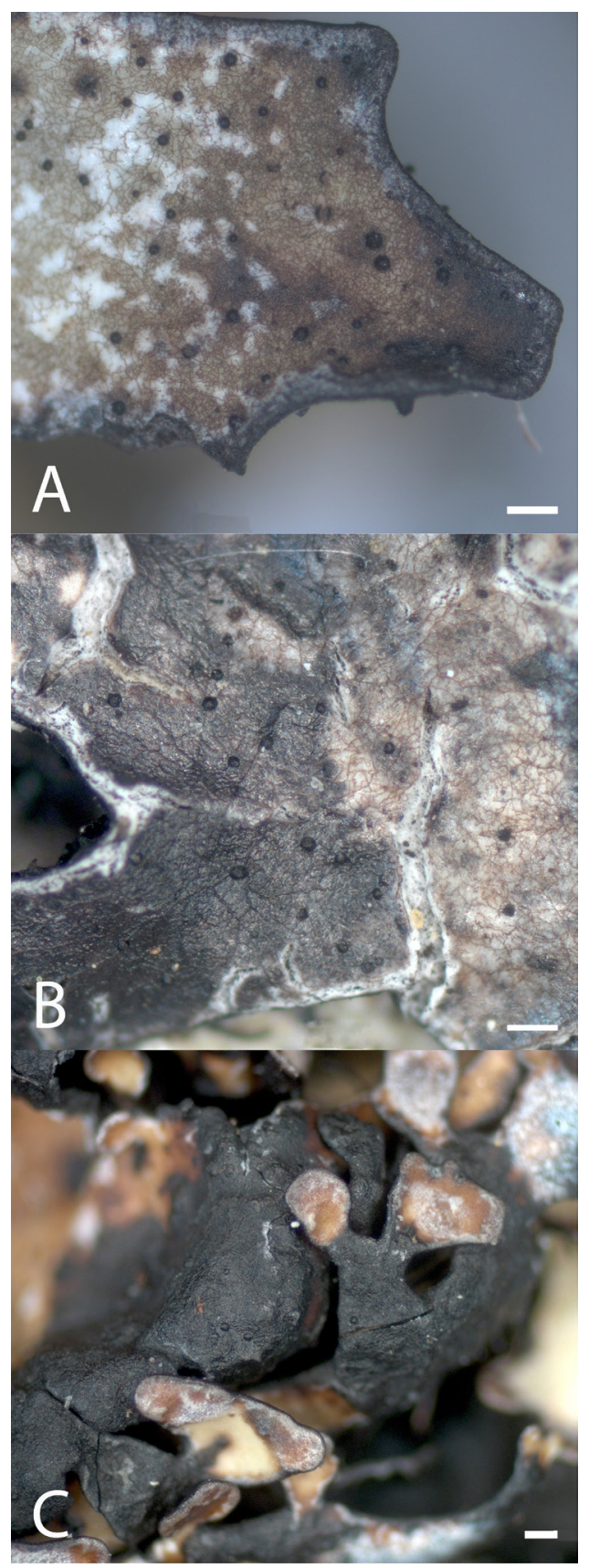

Fig. 1. A: Sphaerellothecium cf. parmeliae growing on healthy-looking lobes of Parmelia omphalodes (LE 264309b). B: S. cf. parmeliae growing on blackened or bleached parts of Parmelia sulcata lobes (LE 261363). C: S. parmeliae growing on black areas of Parmelia omphalodes lobes (233170). Scale bars $=200 \mu \mathrm{m}$. 
Notes - Vegetative hyphae form a superficial dark reticulum, medium brown, 4-10 $\mu \mathrm{m}$ diam., granulate and scaly-areolate. Ascomata black, subglobose, 25-35(-45) $\mu \mathrm{m}$ diam. ( $\mathrm{n}=28)$, sessile; wall medium brown, granulate. Santesson et al. (2004: 194) introduced the new name "Lichenostigma arctoparmeliae" R. Sant. (ined.), which has never been validly published, but may refer to this fungus.

"SphaeropeziA" sp. - Magadan Region, Ola District, Talan Island, $59^{\circ} 18^{\prime} \mathrm{N}, 149^{\circ} 04^{\prime} \mathrm{E}$, alt. 170 $\mathrm{m}$, mountain tundra, on Hypogymnia physodes (thallus) growing on stone, 10.07.2012, E. V. Zheludeva (LE 264289).

Notes - Ascomata cleistohymenial, subglobose to slightly flattened, dark brown or blackish, 200-250 $\mu \mathrm{m}$ diam., opening by a wide pore, surrounded by radially fissured exciple margins, immersed to finally slightly protruding (Fig. 2).
Exciple without hairs, dark brown above, medium to pale brown below, pigmentation granulose and patchy, $\mathrm{K}+$ acquires olivaceous tinge. Ascomatal center I+ orange and partly blue. Paraphyses filiform, apically not swollen, 1-2 $\mu \mathrm{m}$ thick, not or occasionally branched. Mature asci and ascospores not observed. Infection causes distinct bleaching of host thallus. According to the observed characters this probably undescribed lichenicolous ascomycete on Hypogymnia resembles a species of Sphaeropezia Sacc. (Diederich et al., 2002; Baloch et al., 2013). So far no species of cleistohymenial ascomycetes has been reported from Hypogymnia (Lawrey \& Diederich, 2015).

*Spirographa fusisporella (Nyl.) Zahlbr. Magadan Region, Ola District, Magadan Reserve, Studenaya cabin at Yama River, $59^{\circ} 45^{\prime} \mathrm{N}$, $153^{\circ} 34^{\prime} \mathrm{E}$, alt. $70 \mathrm{~m}$, Chosenia-Larix forest, on

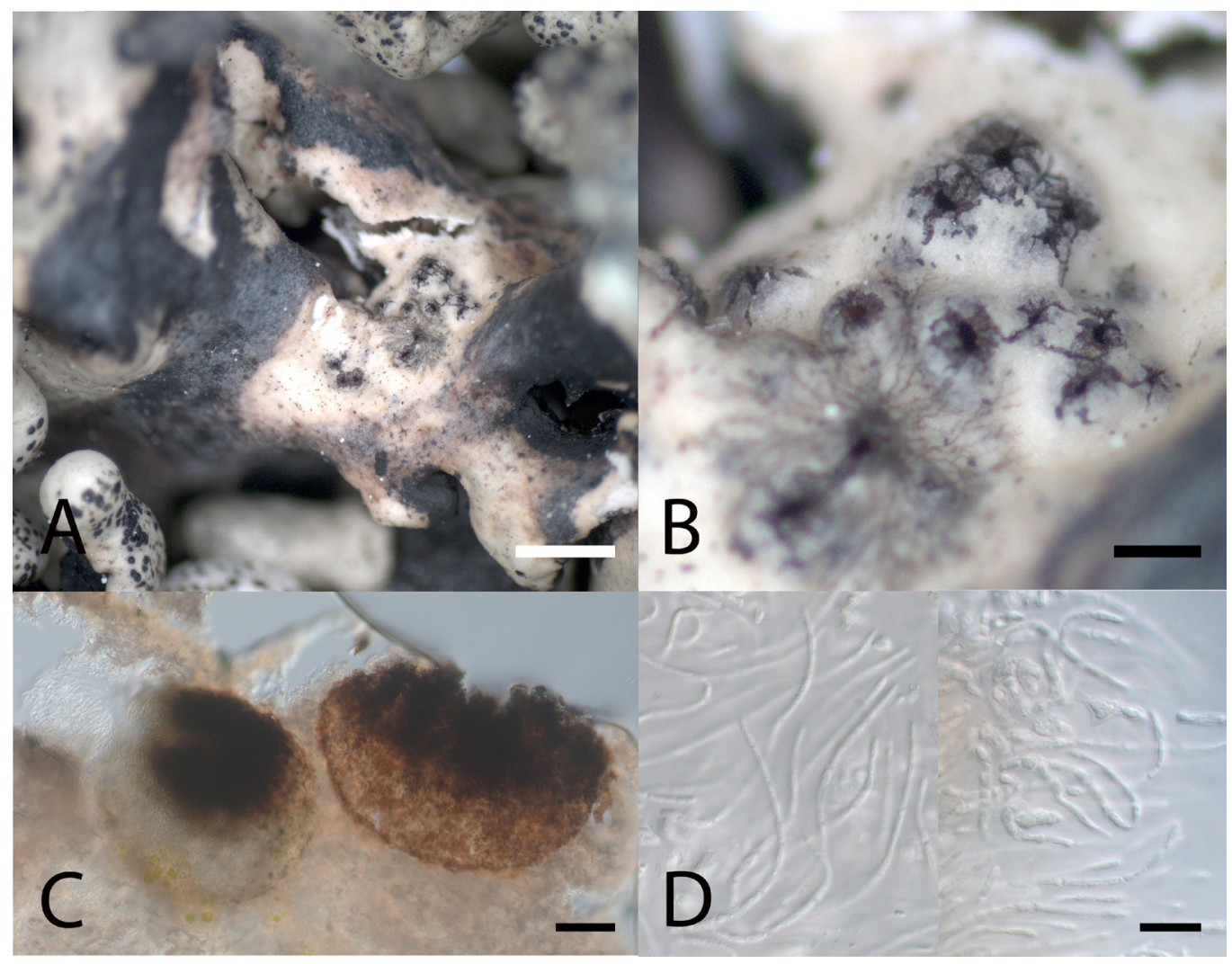

Fig. 2. "Sphaeropezia" sp. on thallus of Hypogymnia physodes (LE 264289). A, B: infection and ascomata habitus. C: squashed young and old ascomata in water. D: paraphyses in water. Scale bars: $A=1 \mathrm{~mm}, \mathrm{~B}=200 \mu \mathrm{m}, \mathrm{C}=50 \mu \mathrm{m}, \mathrm{D}=10 \mu \mathrm{m}$. 
Melanohalea olivacea (damaged hymenium of apothecia), 17.07.2010, E. V. Zheludeva (LE 261461a).

Notes - Formerly known in Russia from Murmansk Region and Komi Republic (Zhurbenko, 2009a; Zhurbenko et al., 2012). The species is widely distributed in the world, but still new to Asia.

*Syzygospora BAChmannil Diederich \& M.S. Christ. - Magadan Region, Ola District, Ola River valley, $59^{\circ} 35^{\prime} \mathrm{N}, 151^{\circ} 15^{\prime} \mathrm{E}$, alt. $15 \mathrm{~m}$, Larix forest, on Cladonia ecmocyna (podetia), 31.07.2009, E. V. Zheludeva (LE 264279).

Notes - Formerly known in Asian Russia from Karachaevo-Cherkesiya Republic, Sverdlovsk Region and Trans-Baikal Territory (Shiryaev et al., 2010; Zhurbenko \& Kobzeva, 2014; Zhurbenko \& Yakovchenko, 2014). So far known in Asia only from Russia.

*Syzygospora PHySCiACEARUm Diederich - Magadan Region, Ola District, Taui River, ichthyological field station, $59^{\circ} 47^{\prime} \mathrm{N}, 148^{\circ} 16^{\prime} \mathrm{E}$, alt. $35 \mathrm{~m}$, Larix-Betula forest, on Physcia aipolia (thallus) growing on Betula, 19.05.2009, E. V. Zheludeva (LE 264369a).

Note - Formerly known in Asian Russia from Republic of Daghestan, Republic of Adygeya and Sverdlovsk Region (Shiryaev et al., 2010; Urbanavichus \& Ismailov, 2013; Urbanavichus \& Urbanavichene, 2014).

\section{ACKNOWLEDGEMENTS}

Paul Diederich, Javier Etayo and Wolfgang von Brackel are thanked for valuable discussions on Sphaerellothecium parmeliae; furthermore, Paul Diederich reviewed the manuscript and made helpful comments. The study of M. P. Zhurbenko was carried out within the framework of the research project of the Komarov Botanical Institute Russian Academy of Sciences no. 01201255602 .

\section{REFERENCES}

Baloch, E., Gilenstam, G. \& Wedin, M. 2013. The relationships of Odontotrema (Odontotremataceae) and the resurrected Sphaeropezia (Stictidaceae)new combinations and three new Sphaeropezia species. Mycologia 105: 384-397. http://dx.doi. org/ $10.3852 / 12-134$
Diederich, P. \& Zhurbenko, M. P. 2009. Sphaerellothecium phaeorrhizae and Zwackhiomyces sipmanii spp. nov. on Phaeorrhiza sareptana from northeastern Asia, with a key to the species of Sphaerellothecium. Bibliotheca Lichenologica 99: 113-122.

Diederich, P., Zhurbenko, M. \& Etayo, J. 2002. The lichenicolous species of Odontotrema (syn. Lethariicola) (Ascomycota, Ostropales). Lichenologist 34: 479-501. http://dx.doi.org/10.1006/ lich. 2002.0418

Esslinger, T. L. 2015. A cumulative checklist for the lichen-forming, lichenicolous and allied fungi of the continental United States and Canada. North Dakota State University. http:/ / www.ndsu.edu/ pubweb/ esslinge/chcklst/chcklst7.htm (First Posted 1 December 1997, Most Recent Version (\#20) 19 April 2015), Fargo, North Dakota.

Etayo, J. \& Diederich, P. 1998. Lichenicolous fungi from the western Pyrenees, France and Spain. IV. Ascomycetes. Lichenologist 30: 103-120. http:/ / dx.doi.org/10.1017/S0024282992000112

Hafellner, J. \& John, V. 2006. Über Funde lichenicoler nicht-lichenisierter Pilze in der Türkei, mit einer Synopsis der bisher im Land nachgewiesenen Taxa. Herzogia 19: 155-176.

Hansen, E. S. \& Alstrup, V. 1995. The lichenicolous fungi on Cladonia subgenus Cladina in Greenland. Graphis Scripta 7(1): 33-38.

Hawksworth, D. L. 1980. Notes on British lichenicolous fungi: III. Notes from the Royal Botanical Garden Edinburgh 38: 165-183.

Kristinsson, H., Zhurbenko, M. \& Hansen E. S. 2010. Panarctic checklist of lichens and lichenicolous fungi. CAFF Technical Report No. 20, CAFF International Secretariat, Akureyri, Iceland. 120 pp.

Lawrey, J. D. \& Diederich, P. 2015. Lichenicolous fungi - worldwide checklist, including isolated cultures and sequences available. http://www. lichenicolous.net [consulted 1.02.2015].

Santesson, R., Moberg, R., Nordin, A., Tønsberg, T. \& Vitikainen, O. 2004. Lichen-forming and lichenicolous fungi of Fennoscandia. Museum of Evolution, Uppsala University. 359 pp.

Shiryaev, A. G., Kotiranta, H., Mukhin, V. A., Stavishenko, I. V. \& Ushakova, N. V. 2010. Aphyllophoroid fungi of Sverdlovsk region: biodiversity, ecology and the IUCN threat categories. Goshchitskiy Publ., Ekaterinburg. 304 pp.

Urbanavichus, G. \& Ismailov, A. 2013. The lichen flora of Gunib plateau, inner-mountain Dagestan (North-East Caucasus, Russia). Turkish Journal of Botany 37: 753-768. http:/ /dx.doi.org/10.3906/ bot-1205-4

Urbanavichus, G. \& Urbanavichene, I. 2014. An inventory of the lichen flora of Lagonaki Highland (NW Caucasus, Russia). Herzogia 27: 285-319. http:/ / dx.doi.org/ 10.13158/heia.27.2.2014.285

Zhurbenko, M. P. 1998. Lichens and lichenicolous fungi from the north of Pyasino lake, Taimyr peninsula, Siberia. Folia Cryptogamica Estonica 32: 153-159. 
Zhurbenko, M. P. 2008. Lichenicolous fungi from Russia, mainly from its Arctic. II. Mycologia Balcanica 5(1-2): 13-22.

Zhurbenko, M. P. 2009a. Lichenicolous fungi and some lichens from the Holarctic. Opuscula Philolichenum 6: 87-120.

Zhurbenko, M. P. 2009b. Lichenicolous fungi and lichens from the Holarctic. Part II. Opuscula Philolichenum 7: 121-186.

Zhurbenko, M. 2009c. Sagediopsis pertusariicola (Verrucariales), a new lichenicolous ascomycete from the Arctic. Nova Hedwigia 88(3-4): 549-555. http://dx.doi.org/10.1127/00295035/2009/0088-0549

Zhurbenko, M. P. 2010. Lichenicolous fungi and lichens growing on Stereocaulon from the Holarctic, with a key to the known species. Opuscula Philolichenum 8: 9-39.

Zhurbenko, M. P. 2012. Lichenicolous fungi growing on Thamnolia, mainly from the Holarctic, with a worldwide key to the known species. Lichenologist 44: 147-177. http://dx.doi.org/10.1017/ S0024282911000739

Zhurbenko, M. P. 2014. Lichenicolous fungi from Far East of Russia. Folia Cryptogamica Estonica 51: 113-119.

Zhurbenko, M. P. \& Braun, U. 2013. Ameroconium cladoniae gen. et sp. nov. and Phoma psoromatis sp. nov., new anamorphic lichenicolous fungi from the Holarctic. Lichenologist 45: 583-591. http:/ / dx.doi.org/10.1017/S0024282913000285

Zhurbenko, M. P. \& Kobzeva, A. A. 2014. Lichenicolous fungi from Northwest Caucasus, Russia. Herzogia 27: 377-396. http://dx.doi.org/10.12697/ fce.2014.51.13 http://dx.doi.org/10.13158/ heia.27.2.2014.377

Zhurbenko, M. P. \& Otnyukova, T. N. 2001. Lichenicolous fungi from the Sayan-Tuva Mountains, Southern Siberia, Russia. Folia Cryptogamica Estonica 38: 79-84.

Zhurbenko, M. P. \& Vershinina, S. E. 2014. Opegrapha bryoriae sp. nov. and other lichenicolous fungi from Asian Russia. Herzogia 27: 93-109. http:/ / dx.doi.org/10.13158/heia.27.1.2014.93

Zhurbenko, M. P. \& Yakovchenko, L. S. 2014. A new species, Sagediopsis vasilyevae, and other lichenicolous fungi from Zabaikal'skii Territory of Russia, southern Siberia. Folia Cryptogamica Estonica 51: 121-130. http://dx.doi.org/10.12697/ fce.2014.51.14

Zhurbenko, M. P., Hermansson, J. \& Pystina, T. N. 2012. Endococcus incrassatus new to Eurasia and some other lichenicolous fungi from the Komi Republic of Russia. Graphis Scripta 24: 36-39. 
108 Folia Cryptog. Estonica 